\title{
Evolution of topological order in Xe films on a quasicrystal surface
}

\author{
Stefano Curtarolo ${ }^{1,3}$, Wahyu Setyawan ${ }^{1}$, Nicola Ferralis $^{2}$, Renee D. Diehl ${ }^{2}$, Milton W. Cole ${ }^{2}$ \\ ${ }^{1}$ Department of Mechanical Engineering and Materials Science, Duke University, Durham, NC 27708 \\ ${ }^{2}$ Department of Physics and Materials Research Institute, Penn State University, University Park, PA 16801 \\ ${ }^{3}$ corresponding author, e-mail: stefano@duke.edu
}

(September 26, 2018)

\begin{abstract}
We report results of the first computer simulation studies of a physically adsorbed gas on a quasicrystalline surface, Xe on decagonal Al-Ni-Co. The grand canonical Monte Carlo method is employed, using a semi-empirical gas-surface interaction, based on conventional combining rules, and the usual Lennard-Jones Xe-Xe interaction. The resulting adsorption isotherms and calculated structures are consistent with the results of LEED experimental data. The evolution of the bulk film begins in the second layer, while the low coverage behavior is epitaxial. This transition from 5-fold to 6-fold ordering is temperature dependent, occurring earlier (at lower coverage) for the higher temperatures.
\end{abstract}

The observed unusual electronic $[1,2]$ and frictional [3-5] properties of quasicrystal surfaces stimulate interesting fundamental questions about how these and other physical properties are altered by quasiperiodicity. Recent progress in the characterization and preparation of quasicrystal surfaces raises new possibilities for their use as substrates in the growth of films having novel structural, electronic, dynamic and mechanical properties [6], or as templates to facilitate the formation of well-ordered and/or size-selected nanoscale aperiodic arrays of clusters or molecules $[7,8]$.

The physical behavior of systems involving competing interactions in adsorption is a subject of continuing interest [9] and is particularly relevant to the growth of thin films. Several different growth modes have been observed for the growth of metal films on quasicrystals. For instance, $\mathrm{Cu}$ on $\mathrm{Al}-\mathrm{Pd}-\mathrm{Mn}$ grows layer-by-layer to produce a film having aperiodic order in one direction [10]. $\mathrm{Al}$ on $\mathrm{Al}-\mathrm{Cu}-\mathrm{Fe}$ forms small size-selected nanoclusters at very low coverages [11]. $\mathrm{Ag}$ on $\mathrm{Al}-\mathrm{Pd}-\mathrm{Mn}$ nucleates at strong-binding sites and ultimately grows as pyramidal hexagonal nanocrystals [12]. Sb and $\mathrm{Bi}$ on $\mathrm{Al}-\mathrm{Pd}-\mathrm{Mn}$ and Al-Ni-Co form quasiperiodic monolayers [13]. Some of these structures convert to different, often periodic, structures when annealed, and the issue of true equilibrium in the film is sometimes complicated by a tendency for chemical intermixing with the substrate. The wide range of behavior observed so far indicates that, even in the absence of intermixing, film growth is strongly affected by chemical interactions between adsorbate and substrate. In order to separate these chemical effects from those specific to quasiperiodic order, we have studied the adsorption of rare gases on a quasicrystal surface, where both the gas-gas and gas-surface interactions are believed to be simple, i.e., appreciable chemical interactions and adsorbate-induced surface reconstructions are absent.

In the present work, we explore the implications of structural mismatch by evaluating the nature of Xe ad- sorption on a quasicrystal substrate, namely the 10-fold surface of decagonal Al-Ni-Co. This study of thermal and structural properties employs grand canonical Monte Carlo (GCMC) simulations, with which we have extensive experience [14-17]. The calculations employ the same potential function we used earlier to compute the low coverage adsorption with the virial expansion [18]. Using the GCMC method, we compute the film properties for specified thermodynamic conditions. A hard wall at $10 \mathrm{~nm}$ above the surface is used to confine the coexisting vapor phase. We take a square section of the surface $A$, of side $5.12 \mathrm{~nm}$, to be the unit cell in the simulation, for which we assume periodic boundary conditions. This approach sacrifices accuracy of the long range QC structure. However, such a simplification is numerically useful for these simulations. Since the cell is large relative to the Xe size it is accurately representative of order on short-to-moderate length scales. The simulation results, presented below, are remarkably consistent with both the results from the virial calculations [18] and with our experiment [19]: a monolayer film is found, the ordering of which reflects many aspects of the underlying quasicrystalline structure. During the onset of second layer adsorption, there is a transition into a 6-fold structure, which after several layers is consistent with a bulk Xe structure.

The Xe-surface potential used here, shown in Figure 1, was calculated earlier by first developing an empirical adsorption potential, based on simple combining rules and known properties of bulk phases of solid $\mathrm{Al}, \mathrm{Ni}$ and $\mathrm{Co}$ crystals [18]. The potential is based on a summation of two-body interactions between the Xe and the individual constituent atoms of the substrate: $\mathrm{Al}, \mathrm{Ni}$ and Co. The gas-gas potential is taken simply to be a LennardJones (LJ) interaction, with parameter values for Xe: $\sigma=221 \mathrm{~K}$ and $\epsilon=0.41 \mathrm{~nm}$. The Xe-substrate pair interactions are also assumed to have LJ form, with parameter values taken from traditional combining rules, using atomic sizes derived from bulk crystalline lattice 
constants [18-20]. In the calculation of the adsorption potential, we assumed a structure of the virgin surface taken from an empirical fit to LEED data [21].

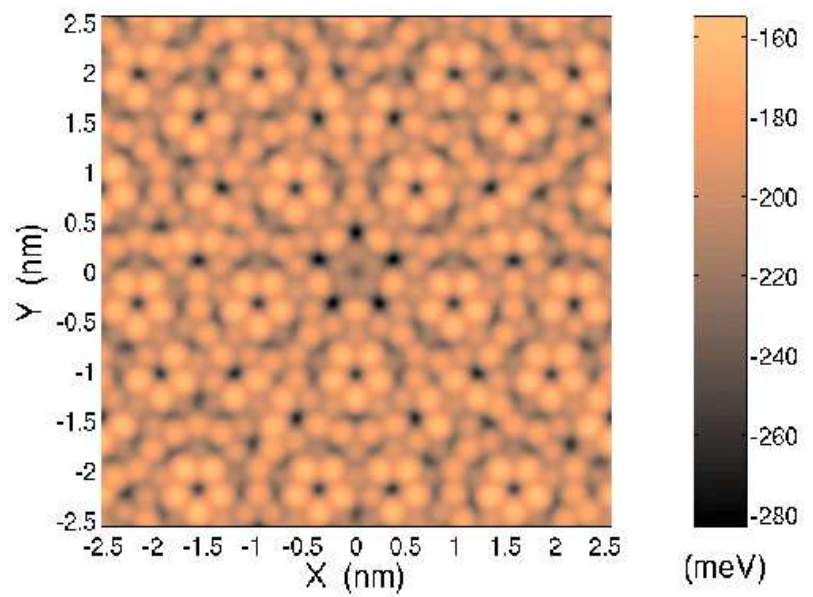

FIG. 1. Computed potential energy for Xe on AlNiCo and potential energy scale (right), obtained by minimizing $V(z, y, z)$ with respect to $z$ variation.

The Xe adsorption potential derived with this procedure is both deep and highly corrugated. Depending on the lateral position $(x, y)$ across the surface, the maximum depth as a function of normal coordinate $(z)$ is typically in the range $D(x, y) \sim 150$ to $250 \mathrm{meV}$. This potential is called "deep" because the record maximum well-depth for Xe on a periodic surface is about $160 \mathrm{meV}$, viz. on graphite [22]; the record minimum well-depth is about $28 \mathrm{meV}$, on $\mathrm{Cs}$ [23]. While the term "corrugation" is not well-defined for a non-periodic surface, one estimate of its magnitude comes from the standard deviation of the laterally varying well-depth, which is about $50 \mathrm{meV}$ on this surface. Thus the well-depth's fluctuation is about $25 \%$ of its average magnitude, which is sufficiently large as to warrant the description "highly corrugated", in our opinion. Using this potential, we computed the adsorption properties at low vapor pressure, $P$, using the virial expansion (including the first three terms). The results of that analysis were found to be semi-quantitatively consistent with our experimental data in the low coverage regime [18].

Figure 2 shows adsorption isotherms computed with the GCMC simulations. The temperature range explored here extends from $70 \mathrm{~K}$ to $286 \mathrm{~K}$ (the triple temperature of Xe is $161.4 \mathrm{~K}$ ). The plotted quantity is the thermodynamic excess coverage, $N_{x} / A$, defined as the difference between the total number of atoms in the simulation cell, and the number that would be present if the cell were filled with uniform vapor at the specified values of $P$ and $T$. Detailed inspection of the isotherms reveals that there is continuous film growth (i.e. complete wetting) for all temperatures above the triple point. This behavior persists to some temperature below the triple temperature but we have not clearly established where the wetting transition occurs. At $77 \mathrm{~K}$, at least 5 layers form before the onset of bulk condensation. The inset in Figure 2 shows the density profile, $\rho(z)$, in the direction perpendicular to the surface, for a total surface coverage of 26.28 atoms $/ \mathrm{nm}^{2}$, corresponding to point "e" on the $77 \mathrm{~K}$ isotherm. One observes that the (perpendicular) layer structure is well defined, in spite of the large lateral variation of the potential.

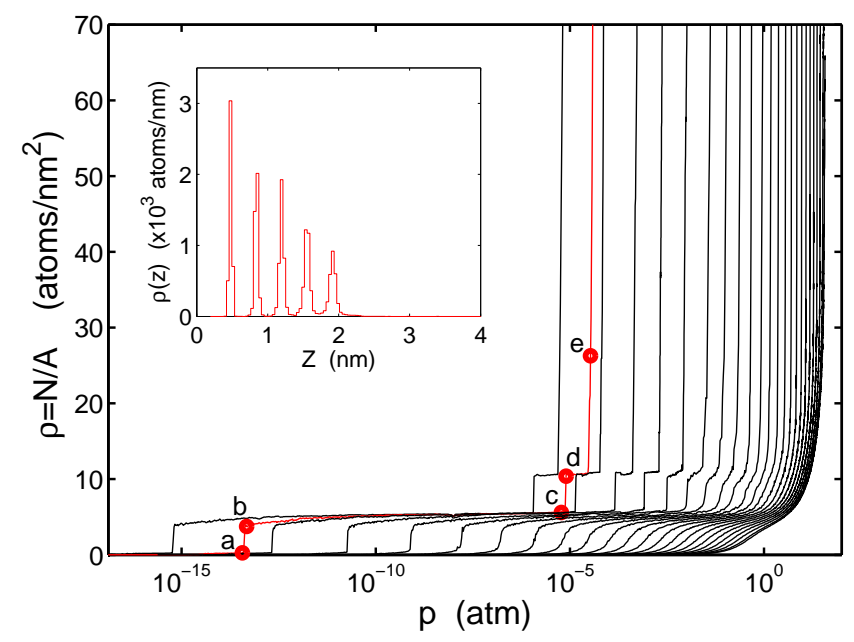

FIG. 2. Computed isotherms from $70 \mathrm{~K}$ to $280 \mathrm{~K}$ in steps of $10 \mathrm{~K}$. Two additional isobars at $77 \mathrm{~K}$ and $286 \mathrm{~K}$ are shown. The isotherm with solid circles is for $77 \mathrm{~K}$, while the highest-temperature isotherm is for $286 \mathrm{~K}$. The inset shows the density profile $\rho(z)$ for $P=0.259 \times 10^{-4}$ atm and $77 \mathrm{~K}$, indicated by point (e).

Figure 3 shows the density variations within the top layer for several points on the $77 \mathrm{~K}$ isotherm as specified in Figure 2. At the lowest pressure (point a), one observes a film that has atoms localized in the deepest parts of the potential, and the Fourier transform (FT) of that density function is 10-fold symmetric, reflecting the substrate symmetry. At the top of the first-layer step of the isotherm (point $b$ ), the density variation is more uniform, now having points of localized 5 -fold and 6 -fold symmetry. The FT of this structure is still 10 -fold, i.e. still reflecting the substrate symmetry. By the time the adsorption proceeds to point $\mathrm{c}$, however, the layer has more points of local 6-fold symmetry, and the FT displays 6 -fold symmetry. When the full bilayer has formed (point d) the in-plane symmetry is clearly 6-fold locally, with some dislocation defects, and the FT indicates an overall 6 -fold symmetry of the structure. When the 6 -fold structure forms, it is aligned so that the close-packed direction of the $\mathrm{Xe}$ is parallel to a principal 5 -fold direction of the substrate surface. For any given simulation run, the selection of 5-fold/6-fold alignment is arbitrary, and in Figure 3d, it can be seen that there are actually two alignments present.

Xe adsorption on this surface was studied earlier using low-energy electron diffraction, and isobar measurements indicate that the Xe film grows layer-by-layer in the tem- 
perature range $65 \mathrm{~K}$ to $80 \mathrm{~K}[19]$, consistent with the simulations described above. Figure 3 shows the LEED patterns obtained from adsorbed Xe on decagonal Al-Ni-Co under similar conditions to the simulations. At the lowest coverage, the only discernible change in the LEED pattern from that of the clean surface is an attenuation of the substrate beams. After the adsorption of one layer (coverage determined by the isobar measurements [19]) there are still no resolvable features that would indicate an overlayer having order different from the substrate. At the onset of the adsorption of the second layer, however, the LEED pattern shows new diffraction spots that correspond to 5 rotational domains of a hexagonal structure. Within each of these domains, the close-packed direction of the $\mathrm{Xe}$ is aligned with the 5-fold directions of the substrate [19], as observed in the simulation. In the experiments, all possible alignments are observed owing to the presence of all possible rotational alignments present within the width of the electron beam $(0.25 \mathrm{~mm})$, and there is no evidence of step-pinning of the overlayer. When the second layer is complete, these spots are welldefined and their widths are the same as the substrate spots, indicating a coherence length of at least $15 \mathrm{~nm}$. A dynamical LEED analysis of the intensities indicates that the structure of the multilayer film is consistent with fcc $\mathrm{Xe}(111)$.

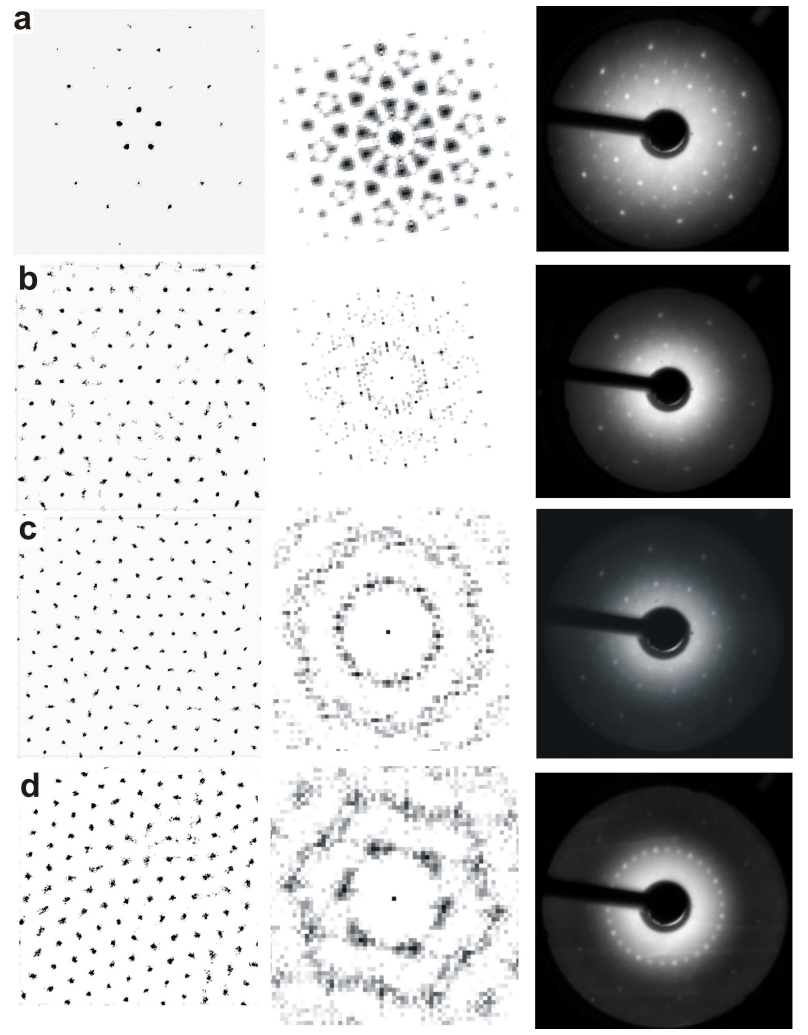

FIG. 3. Intralayer density plots for adsorption at $77 \mathrm{~K}$, Fourier transforms of these plots and LEED patterns corresponding to similar conditions. The plots show the density in the top layer, corresponding to the points noted in Figure 2.
While the experiments are restricted to a comparatively narrow range in $T$ and $P$ (the LEED experiments require $P<10^{-7}$ bar and feasible equilibration times require $P>10^{-13}$ bar) the simulations are not so restricted. Therefore, we have also investigated the adsorption of Xe at a very low temperature $(20 \mathrm{~K})$ and a high temperature $(160 \mathrm{~K})$. The density plots and FT's at the coverage corresponding to point $\mathrm{c}$ in Figure 3 is shown on Figure 4 for the three temperatures, $20 \mathrm{~K}, 77$ $\mathrm{K}$ and $160 \mathrm{~K}$. The trend observed in the ordering is that the onset of 6 -fold ordering occurs earlier at the higher temperatures. Six-fold ordering is already present at this coverage for $160 \mathrm{~K}$, but is barely present at $77 \mathrm{~K}$ and is clearly not present at $20 \mathrm{~K}$. This trend is consistent with the substrate potential being a bigger influence on the film's structure at lower $T$, implying that the development of the bulk Xe structure is entropy-driven; this is a kind of wetting transition of the bulk Xe phase, attributable to a diminishing interfacial free energy cost with increasing $T$. More thermal disorder is also evident in the film structures at higher $T$.

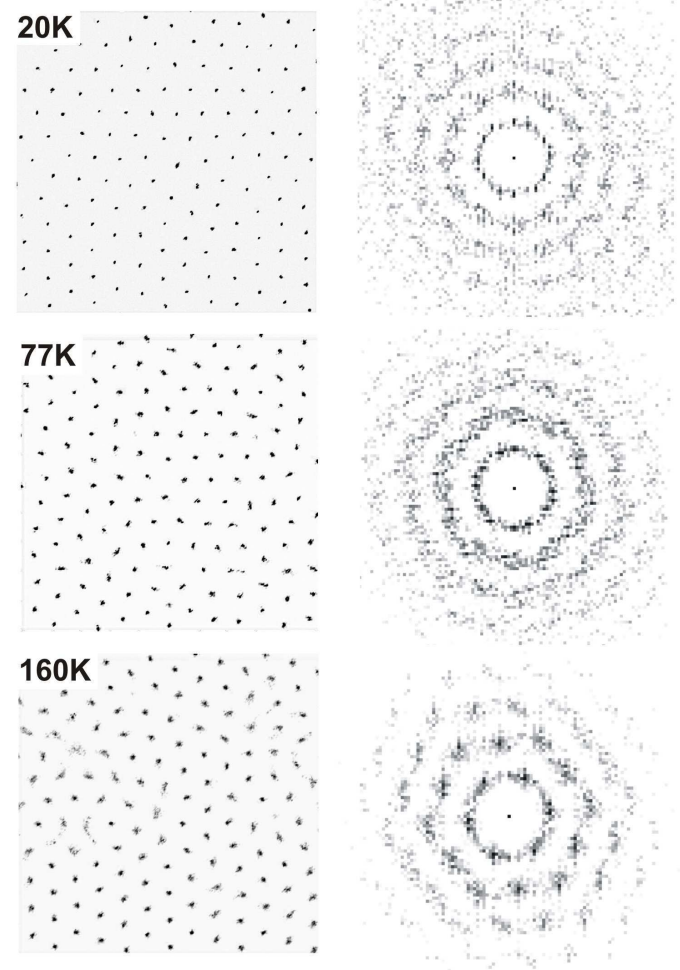

FIG. 4. Density plots and Fourier transforms for monolayer films (corresponding to point $\mathrm{c}$ in Figure 2) at $20 \mathrm{~K}, 77 \mathrm{~K}$, and $160 \mathrm{~K}$.

Interestingly, at all $T$ studied, stacking faults are evident in the multilayer films. Their origin appears to be dislocations in the layers, which are most prevalent at the highest temperatures studied, as expected for entropic reasons. This is consistent with $\mathrm{x}$-ray diffraction studies of the growth of Xe on $\operatorname{Ag}(111)$, where stacking faults 
were observed for Xe growth under various growth conditions [24,25], although the overall structure observed was fcc(111). Such a stacking fault is evident in Figure 5, which shows a superposition of Xe layers 2 and 4 at point e in Figure 2. The coincidence of the atom locations in the top left part of this figure is consistent with an hcp structure (ABAB stacking) whereas the offset observed in the lower left is consistent with an fcc structure (ABC stacking). We note that while bulk Xe has an fcc structure, and indeed an fcc structure was found for the multilayer film in the LEED study, calculations of the bulk structure using LJ pair potentials such as those employed here result in a more stable hcp structure [26]. This difference apparently arises from a neglect (using the pair potentials) of d-orbital overlap interactions, which are more effective in fcc than in hcp structures [26]. This model agrees quantitatively with measurements of energy differences in fcc and hcp Ar near its triple point [27]. The presence of dislocations and disclinations in the monolayer films and their variation with temperature raises interesting questions concerning the possibility of dislocation-mediated melting [28-30], but the limited simulation size precludes any such analysis. Larger simulations with Xe and different noble gases are intended for the future.

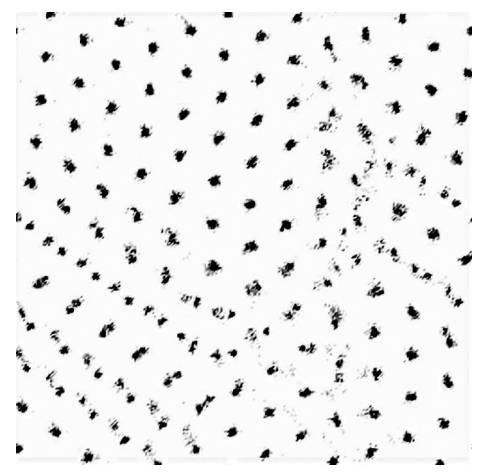

FIG. 5. Density plot corresponding to point c on Figure 2 , showing a superposition of the density slices for the $2^{\text {nd }}$ and $4^{t h}$ layers. In the top right, $4^{\text {th }}$-layer atoms are located directly above the $2^{n d}$ layer atoms, whereas in other regions, such as lower left, the two layers are offset.

In summary, our simulation results are consistent with the experimental data over the somewhat limited range explored thus far. A consistent pattern has emerged; the quasicrystalline order is transmitted to films of thickness less than about two layers, disappearing upon further growth of the film. The results of this study encourage confidence in the use of physical adsorption to probe film growth. The extension of these calculations to the study of other properties such as lattice dynamics and friction, as well as other adsorbates, would seem well justified. Such studies will yield a comprehensive understanding of competing interactions in physisorbed layers on aperiodic substrates, as found previously on periodic surfaces.

This research was supported by NSF grant DMR0208520. We wish to acknowledge helpful discussions with L. W. Bruch, Jorge Sofo, Mike Widom, Aleksey Kolmogorov, and R. Andreea Trasca.

[1] E. Rotenberg, W. Theis, K. Horn, and P. Gille, Nature 406, $602(2000)$

[2] E. Rotenberg, W. Theis, and K. Horn, J. Alloys Compd. 342, 348 (2002).

[3] J. M. Dubois, Physica Scripta T49, 17 (1993).

[4] J. S. Ko, A. J. Gellman, T. A. Lograsso, C. J. Jenks, and P. A. Thiel, Surf. Sci. 423, 243 (1998).

[5] J. Y. Park, D. F. Ogletree, M. Salmeron, C. J. Jenks, and P. A. Thiel, Tribology Letters 17, 629 (2004).

[6] R. McGrath, U. Grimm, and R. D. Diehl, Physics World 17, 23 (2004).

[7] R. McGrath, J. Ledieu, E. J. Cox, and R. D. Diehl, J. Phys.: Condensed Matter 14, R119 (2002).

[8] R. McGrath, J. Ledieu, E. J. Cox, S. Haq, R. D. Diehl, C. J. Jenks, I. Fisher, A. R. Ross, and T. A. Lograsso, J. Alloys Compd. 342, 432 (2002).

[9] L. W. Bruch, M. W. Cole, and E. Zaremba, Physical Adsorption: Forces and Phenomena (Oxford University Press, Oxford, 1997).

[10] J. Ledieu, J.-T. Hoeft, D. E. Reid, J. Smerdon, R. D. Diehl, T. A. Lograsso, A. R. Ross, and R. McGrath, Phys. Rev. Lett. 92, 135507 (2004).

[11] T. Cai, J. Ledieu, V. Fournee, T. Lograsso, A. Ross, R. McGrath, and P. A. Thiel, Surf. Sci. 526, 115 (2003).

[12] V. Fournee, T. C. Cai, A. R. Ross, T. A. Lograsso, J. W. Evans, and P. A. Thiel, Physical Review B 67, 033406 (2003).

[13] K. J. Franke, H. R. Sharma, W. Theis, P. Gille, P. Ebert, and K. H. Rieder, Phys. Rev. Lett. 89, 156104 (2002).

[14] F. Ancilotto, S. Curtarolo, F. Toigo, and M. W. Cole, Phys. Rev. Lett. 87, 206103 (2001).

[15] S. Curtarolo, G. Stan, M. W. Cole, M. J. Bojan, and W. A. Steele, Phys. Rev. E 59, 4402 (1999).

[16] S. Curtarolo, G. Stan, M. J. Bojan, M. W. Cole, and W. A. Steele, Phys. Rev. E 61, 1670 (2000).

[17] S. Curtarolo, M. W. Cole, and R. D. Diehl, Phys. Rev. B 70, 115403 (2004).

[18] R. A. Trasca, N. Ferralis, R. D. Diehl, and M. W. Cole, J. Phys: Condens. Mat. 16, S2911 (2004).

[19] N. Ferralis, R. D. Diehl, K. Pussi, M. Lindroos, I. R. Fisher, and C. J. Jenks, Phys. Rev. B 69, 075410 (2004).

[20] A similar approach was used earlier to describe the interaction of $\mathrm{Al}$ on quasicrystalline $\mathrm{AlNiCo}$; see T. Fluckiger, Y. Weisskopf, M. Erbudak, R. Luscher, and A. R. Kortan, Nano Letters 3, 1717 (2003).

[21] N. Ferralis, K. Pussi, E. J. Cox, M. Gierer, J. Ledieu, I. R. Fisher, C. J. Jenks, M. Lindroos, R. McGrath, and R. D. Diehl, Phys. Rev. B 69, 153404 (2003).

[22] G. Vidali, G. Ihm, H.-Y. Kim, and M. W. Cole, Surf. Sci. Reports 12, 133 (1991).

[23] A. Chizmeshya, M. W. Cole, and E. Zaremba, J. Low Temp. Phys. 110, 677 (1998)

[24] P. Dai, T. Angot, S. N. Ehrlich, S.-K. Wang, and H. Taub, Phys. Rev. Lett. 72, 685 (1994).

[25] P. Dai, Z. Wu, T. Angot, S.-K. Wang, H. Taub, and S. N. Ehrlich, Phys. Rev. B 59, 15464 (1999).

[26] K. F. Niebel and J. A. Venables, in Rare Gas Solids, edited by M. L. Klein and J. A. Venables (Academic Press, New York, 1976), Vol. 1, p. 558.

[27] T. Bricheno and J. A. Venables, J. Phys. C: Solid State Physics 9 4095, (1976).

[28] J. M. Kosterlitz and D. J. Thouless, J. Phys. C: Solid State Physics 6, 1181 (1972).

[29] D. R. Nelson and B. I. Halperin, Phys. Rev. B 19, 2457 (1979).

[30] A. P. Young, Phys. Rev. B 19, 1855 (1979). 